\title{
Randomized trial of subfascial infusion of ropivacaine for early recovery in laparoscopic colorectal cancer surgery
}

\author{
Sang Hyun Lee ${ }^{1}$, Woo-Seog Sim ${ }^{1}$, Go Eun Kim ${ }^{1}$, Hee Cheol Kim², \\ Joo Hyun Jun ${ }^{3}$, Jin Young Lee ${ }^{1}$, Byung-Seop Shin ${ }^{1}$, Heejin Yoo ${ }^{4}$, Sin-Ho Jung ${ }^{4}$, \\ Joungyoun $\mathrm{Kim}^{5}$, Seung Hyeon Lee ${ }^{1}$, Deok Kyu $\mathrm{Yo}^{1}$, and $\mathrm{Yu} \mathrm{Ri} \mathrm{Na}^{1}$ \\ ${ }^{1}$ Department of Anesthesiology and Pain Medicine, ${ }^{2}$ Division of Colorectal Surgery, Samsung Medical Center, \\ Sungkyunkwan University School of Medicine, ${ }^{3}$ Department of Anesthesiology and Pain Medicine, Kangnam Sacred \\ Heart Hospital, Hallym University of College of Medicine, ${ }^{4}$ Biostatistics and Clinical Epidemiology Center, Samsung \\ Medical Center, Seoul, ${ }^{5}$ Department of Information Statistics, Chungbuk National University, Cheongju, Korea
}

Background: There is a need for investigating the analgesic method as part of early recovery after surgery tailored for laparoscopic colorectal cancer (LCRC) surgery. In this randomized trial, we aimed to investigate the analgesic efficacy of an inverse "v" shaped bilateral, subfascial ropivacaine continuous infusion in LCRC surgery.

Methods: Forty two patients undergoing elective LCRC surgery were randomly allocated to one of two groups to receive either $0.5 \%$ ropivacaine continuous infusion at the subfascial plane $(\mathrm{n}=20$, R group) or fentanyl intravenous patient controlled analgesia (IV PCA) $(n=22, \mathrm{~F}$ group) for postoperative 72 hours. The primary endpoint was the visual analogue scores (VAS) when coughing at postoperative 24 hours. Secondary end points were the VAS at 1, 6, 48, and 72 hours, time to first flatus, time to first rescue meperidine requirement, rescue meperidine consumption, length of hospital stay, postoperative nausea and vomiting, sedation, hypotension, dizziness, headache, and wound complications.

Results: The VAS at rest and when coughing were similar between the groups throughout the study. The time to first gas passage and time to first rescue meperidine at ward were significantly shorter in the R group compared to the F group ( $\mathrm{P}$ $=0.010)$. Rescue meperidine was administered less in the R group; however, without statistical significance. Other study parameters were not different between the groups.

Conclusions: Ropivacaine continuous infusion with an inverse "v" shaped bilateral, subfascial catheter placement showed significantly enhanced bowel recovery and analgesic efficacy was not different from IV PCA in LCRC surgery.

Key Words: Analgesia, Colorectal surgery, Laparoscopy, Local anesthetics, Ropivacaine, Subfascia.

Corresponding author: Woo-Seog Sim, M.D., Ph.D.

Department of Anesthesiology and Pain Medicine, Samsung Medical Center, Sungkyunkwan University School of Medicine, 81, Irwon-ro, Gangnam-gu, Seoul 06351, Korea

Tel: 82-2-3410-2471, Fax: 82-2-3410-0361

Email: anesthe@skku.edu

ORCID: http://orcid.org/0000-0002-5346-6424

Received: June 14, 2016.

Revised: August 6, 2016.

Accepted: August 16, 2016.

Korean J Anesthesiol 2016 December 69(6): 604-613 https://doi.org/10.4097/kjae.2016.69.6.604

\section{Introduction}

Laparoscopic colorectal cancer (LCRC) surgery in conjunction with other fast track modalities is increasingly practiced within an early recovery after surgery (ERAS) pathway [1-5]. Strategic, multimodal pain management plays an important part in the ERAS pathway. The analgesic method in LCRC surgery within ERAS had been adopted initially from that of the open colocrectal cancer (CRC) surgical protocol. Therefore, pain management needed to be tailored to LCRC surgeries and its ef-

(c) This is an open-access article distributed under the terms of the Creative Commons Attribution Non-Commercial License (http://creativecommons.org/ licenses/by-nc/4.0/), which permits unrestricted non-commercial use, distribution, and reproduction in any medium, provided the original work is properly cited. 
ficacy needed to be validated [6]. In this regard, local anesthetic infiltration, intravenous patient controlled analgesia (IV PCA), non-steroidal anti-inflammatory drugs or paracetamol, intravenous lidocaine infusion, and neuraxial block have been investigated [5,7-13].

While there is no consensus on the best method of analgesia $[6,8]$, recent studies report that epidural analgesia does not have a beneficial outcome in LCRC surgery and could even be deleterious [11,14,15]. According to Levi et al. [11], return of bowel function was slower and the length of hospital stay and duration of nausea were longer with epidural analgesia compared to spinal anesthesia and IV PCA in LCRC surgery. They concluded that epidural analgesia may not be a good choice for postoperative pain management in LCRC surgery, unlike in open CRC surgery [11]. On the other hand, local anesthetic infiltration techniques, including continuous wound infusion $[9,12,16]$ and transversus abdominis plane (TAP) block [17], provide safe and comparable analgesia as compared to epidural analgesia. In the meantime, IV PCA is also a commonly used analgesia in LCRC surgery, as is in our institution. IV PCA may be considered sufficient to control postoperative pain, because LCRC surgery is relatively less invasive and reportedly provokes less postoperative pain than open CRC surgery [18]. However, systemic opioid IV PCA may accompany opioid side effects, such as nausea and vomiting, pruritus and postoperative ileus, even with the small doses required for analgesia.

Two randomized controlled trials in LCRC surgeries found that when local anesthetic wound infusion were compared to either saline (placebo) wound infusion or epidural analgesia the analgesic effect was comparable [12,19]. Studies of local anesthetics wound infusion in open laparotomy surgeries report controversial results and suggest that success lies in the appropriate plane of wound catheter placement $[20,21]$. In the former two studies in LCRC surgeries, wound catheters were placed at the extraction wound of the specimen $[12,19]$. In comparison, we used a method of placing the catheters at the subfascial layer using laparoscopic guidance and compared this method of subfascial ropivacaine continuous infusion to IV PCA. The innervation of the nerves in the abdomen arises from the thoracic intercostal nerves T6-12, L1, which course through between the internal oblique and the transversus abdominis muscle at the lateral side and converge toward the preperitoneal space at the midline. With these nerve trajectories in mind, we thought that subfascial catheter placement after laparoscopic surgery might be beneficial for postoperative surgical pain, because along with incisional pain, residual abdominal distension from carbon dioxide insufflation and pneumoperitoneum in laparoscopic surgery might trigger nociception in the whole peritoneum and might cause diffuse abdominal pain after surgery [22].

In this randomized clinical trial, we aimed to investigate the analgesic efficacy of this novel method of subfascial ropivacaine continuous infusion in LCRC surgery, and investigated, in regard to ERAS, the time to first flatus, time to first rescue meperidine requirement, rescue meperidine consumption, and length of hospital stay (LOS). Postoperative side effects, including postoperative nausea and vomiting, sedation, hypotension, dizziness, headache, and wound complications were also studied.

\section{Materials and Methods}

This prospective, randomized trial was performed after approval from this Institution's Review Board of ethical committee (IRB file no. 2010-10-011) and observed ethical standards of the Helsinki Declaration. We received an informed consent from all patients. The study protocol was prospectively registered with the Australia and New Zealand Clinical Trial Registry: ACTRN 12611000183987. This study conforms to the CONSORT guideline.

Patients aged between 20-65 years undergoing laparoscopic colorectal surgeries at our single institution between June 2011 and April 2013, who agreed to participate, were included in this study. Patients who were allergic to study drugs, with pre-existing coagulopathy, neurologic or cognitive dysfunction, systemic or regional infection, previously taking opioids for chronic pain, compromised understanding of patient-controlled analgesia, obesity (body mass index $>30 \mathrm{~kg} / \mathrm{m}^{2}$ ), and pregnancy were excluded from the study. Patients were randomly allocated following a simple randomization procedure to one of two parallel groups in $1: 1$ ratio to receive subfascial ropivacaine continuous infusion ( $\mathrm{R}$ group) or fentanyl IV PCA (F group). A random sequence was generated by computer-generated allocation numbering (www.randomizer.org) and allocation concealment was achieved through sequentially numbered concealed sheets. Blinding of the operator was not applied in this study because $\mathrm{R}$ group patients received wound catheters and $\mathrm{F}$ group patients did not. In an attempt to reduce the risk of bias, the research investigator who recorded the postoperative study parameters was not informed of and was oblivious to the presence of the ropivacaine infusion device. However, blinding was not complete in this study.

Patients were anesthetized with thiopental $5 \mathrm{mg} / \mathrm{kg}$, sevoflurane, and rocuronium or vecuronium. After tracheal intubation, anesthesia was maintained with sevoflurane 2 vol\% at $0.5 \mathrm{frac}-$ tion of inspired oxygen with air. Nitrous oxide was not used. Intraoperative analgesia was performed using IV fentanyl. All patients received hydromorphone $0.015 \mathrm{mg} / \mathrm{kg}$ at the end of surgery. In the postanesthetic care unit (PACU) IV meperidine $0.5 \mathrm{mg} / \mathrm{kg}$ was given as a rescue analgesic and IV ketorolac 30 mg was given as the next rescue analgesics. The visual analogue scale (VAS) at discharge from the PACU was equalized to VAS < 
$40 \mathrm{~mm}$ according to PACU discharge criteria.

All surgical procedures and insertion of continuous infusion devices were conducted by one surgeon. Laparoscopic surgical procedures were performed with one peri- or trans-umbilical camera port and 2-3 mini-incisional laparoscopic ports. The specimen was extracted either from the incisional extension from the umbilical port site or from the left lower quadrant incisional wound, ranging 4-6 centimeters in size.

In the $\mathrm{R}$ group, a bolus of $0.5 \%$ ropivacaine $10 \mathrm{ml}$ was injected and a continuous infusion was applied through an elastometric pump (On-Q Painbuster ${ }^{\mathrm{TM}}$, I-Flow Corp., Lake Forest, CA, USA). The pump was preset to deliver $0.5 \%$ ropivacaine through two multi-holed catheters at a rate of $2 \mathrm{ml} / \mathrm{h}$ per catheter during postoperative 72 hours (a total volume of $300 \mathrm{ml}$, infusion rate of $2+2 \mathrm{ml} / \mathrm{h}$ per catheter, $12.5 \mathrm{~cm}$ catheter length). The technique of catheter placement was as follows: a tunneling of the catheters was made from 1 inch below the xyphoid process along the bilateral subcostal margin in the shape of an inverse " $v$ ", with a catheter on each side placed at the subfascial layer, deep to the transversalis fascia (between the parietal peritoneum and the musculo-fascial layer). The catheters were placed by one surgeon under viewing of the laparoscopic images (Fig. 1). In the F group, a fentanyl IV PCA pump (AutoMed ${ }^{\circledR} 3200$, Ace Medical, Korea) was initiated at the PACU and was maintained for postoperative 72 hours. The regimen comprised of $15 \mu \mathrm{g} / \mathrm{ml}$ fentanyl in normal saline $100 \mathrm{ml}$ at an infusion rate of basal $0.5 \mathrm{ml} / \mathrm{h}$, a bolus of $1 \mathrm{ml}$ with a lock out interval 15 minutes.

Postoperative checkups included pain scores recorded by the VAS (VAS, scale of $0-100 \mathrm{~mm}, 0=$ no pain, $100=$ extreme pain) at rest and when coughing, postoperative analgesic requirements, postoperative nausea and vomiting (PONV), sedation, hypotension, dizziness, headache, and wound complications at 1 (at PACU), 6, 24, 48, and 72 hours postoperatively. Time to first flatus, time to first rescue, IV meperidine requirement, and length of hospital stay (LOS) were also recorded.

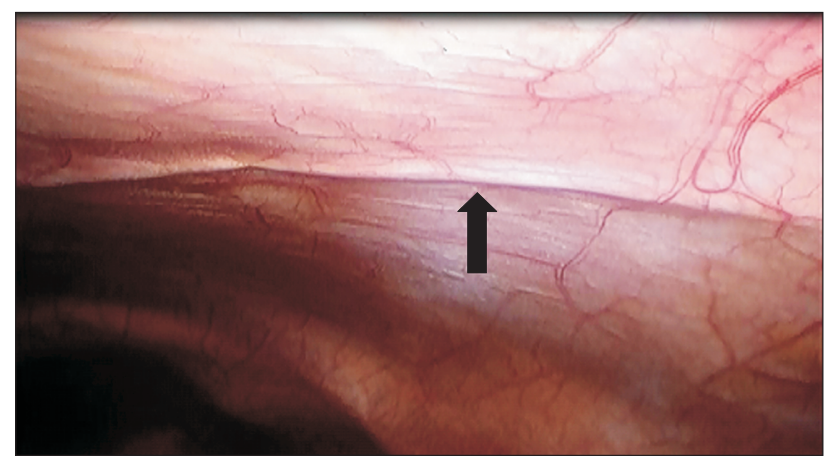

Fig. 1. Laparoscopically assisted ropivacaine continuous infusion (Painbuster ${ }^{\mathrm{TM}}$ ) catheter placement at subfascial layer (preperitoneum). Arrow indicates a catheter placed just beneath the parietal peritoneum.
For effective postoperative analgesia, all patients were made feasible to use a bolus of fentanyl IV PCA at bedside, by equipping the patients in $\mathrm{R}$ group with a bolus only fentanyl IV PCA device (AutoMed ${ }^{\circledR} 3200$, Ace Medical, Seoul, Korea), which was set to deliver a bolus of $15 \mu \mathrm{g}$ fentanyl only at patient's demand (15 $\mu \mathrm{g} / \mathrm{ml}$ of fentanyl in $100 \mathrm{ml}$ normal saline, at lock out interval of 15 minutes). Patients in the F group used their fentanyl IV PCA for bolus demand. All patients received meperidine $0.5 \mathrm{mg} / \mathrm{kg}$ for rescue analgesia if VAS $>40 \mathrm{~mm}$ even after IV PCA bolus. Meperidine was chosen for rescue analgesia according to our hospital's analgesia prescription. Oral analgesia on a regular basis was withheld for postoperative 72 hours during the study period.

Antiemetic drugs were given if PONV was moderate or severe (PONV was questioned on a scale of $10 ; 1-3$ : mild; 4-6: moderate; 7-10: severe). At PACU, IV metoclopramide $10 \mathrm{mg}$ was given as a primary antiemetic drug, and IV palonosetron $0.075 \mathrm{mg}$ was given as a second line drug. At ward, IV metoclopramide $10 \mathrm{mg}$ or granisetron $1 \mathrm{mg}$ was given. Sedation was recorded on a scale of 5 (1: completely awake, 2: drowsy, 3: dozing, 4: mostly sleepy, 5: not responding). Hypotension was recorded if systolic blood pressure $<30 \%$ of baseline. The presence of dizziness was also recorded. The wound was dressed on postoperative day 3 and its condition was made note on the electronic medical record.

\section{Statistics}

A pilot study showed that the VAS when coughing at postoperative 24 hours in IV PCA was $60 \pm 20 \mathrm{~mm}$. Based on the reduction of VAS $>30 \%$ perceived to be clinically relevant $(80 \%$ power test and alpha level of 0.05), 21 patients in each group were required. Accounting the $10 \%$ drop rate, a total of 46 (23 patients each) patients were planned.

Demographic and surgical data, the VAS scores, meperidine and fentanyl IV PCA consumption, resumption of oral soft diet, and satisfaction scores were analyzed by t-test or Wilcoxon rank sum test according to normality test results. Cumulative meperidine consumption was analyzed after natural logarithm transformation due to non-normality. The length of hospital stay, time to first gas out, time to first meperidine requirement at ward, and time to first meperidine requirement including PACU were analyzed by log rank test. Categorical variables including PONV, use of antiemetic drugs, sedation and percentage of patients with no rescue meperidine during postoperative 72 hours were analyzed by chi square or Fisher's exact test, as appropriate. Continuous variables were displayed as mean \pm SD or median (interquartile range) after normality test. All values were considered significant if $\mathrm{P}<0.05$. Statistical analysis was carried out using SAS version 9.3 (SAS Institute Inc, Cary, NC, USA). 


\section{Results}

A total of 44 patients were recruited. Among these 44, one patient refused to participate and one patient was lost during follow up. Thus, 42 patients completed the study (Fig. 2, CONSORT diagram). Patient demographics and details of surgery are listed in Table 1. No significant differences were observed between the groups.

Our primary end point, the VAS when coughing at 24 hours, showed no difference between the groups. The VAS scores at rest and when coughing at all other time points were also not different between the groups (Table 2). The time to first flatus was significantly shorter in the $\mathrm{R}$ group compared to the $\mathrm{F}$ group ( $\mathrm{P}$ $=0.010$ ) (Table 3 ). Resumption of oral soft diet was similar between the groups $(\mathrm{P}=0.492)$. LOS was not different between the groups $(\mathrm{P}=0.853)$.

Analgesic consumption of IV fentanyl, rescue IV meperidine, the number (\%) of patients with no request for rescue IV meperidine during 72 hours postoperatively, and time to first rescue meperidine are displayed in Table 4. Rescue IV meperidine requirement was not different between the groups. IV fentanyl consumption was significantly less in the R group, because of the given protocol (Fentanyl IV PCA group with continuous and bolus doses vs. ropicaine continuous infusion group with fentanyl IV PCA bolus only doses). Time to first meperidine given in the ward was significantly shorter in the $R$ group $(P=0.017)$, but the number of patients who did not request rescue IV meperidine during the 72 hour follow up was smaller in the R group, although this difference was statistically not significant. Rescue IV ketorolac at the PACU was given to two patients in the F group.

Adverse effects, including PONV and sedation were similar between the groups. The incidence of PONV, the use of antiemetic drug, and sedation are shown in Table 5. Antiemetic drugs were given to moderate to severe PONV patients. However, at postoperative $1-6$ hours in the ward, 18 patients (13 in F group, 5 in $\mathrm{R}$ group) received routine IV antiemetic drug, granisetron $1 \mathrm{mg}$ postoperatively. There was no report of hypotension, dizziness, or headache in this study.

There was no mortality or morbidity, nor was there any wound infection or complication related to the analgesic method. One patient in the F group showed a leakage in anastomosis site and received reoperation without further complications.

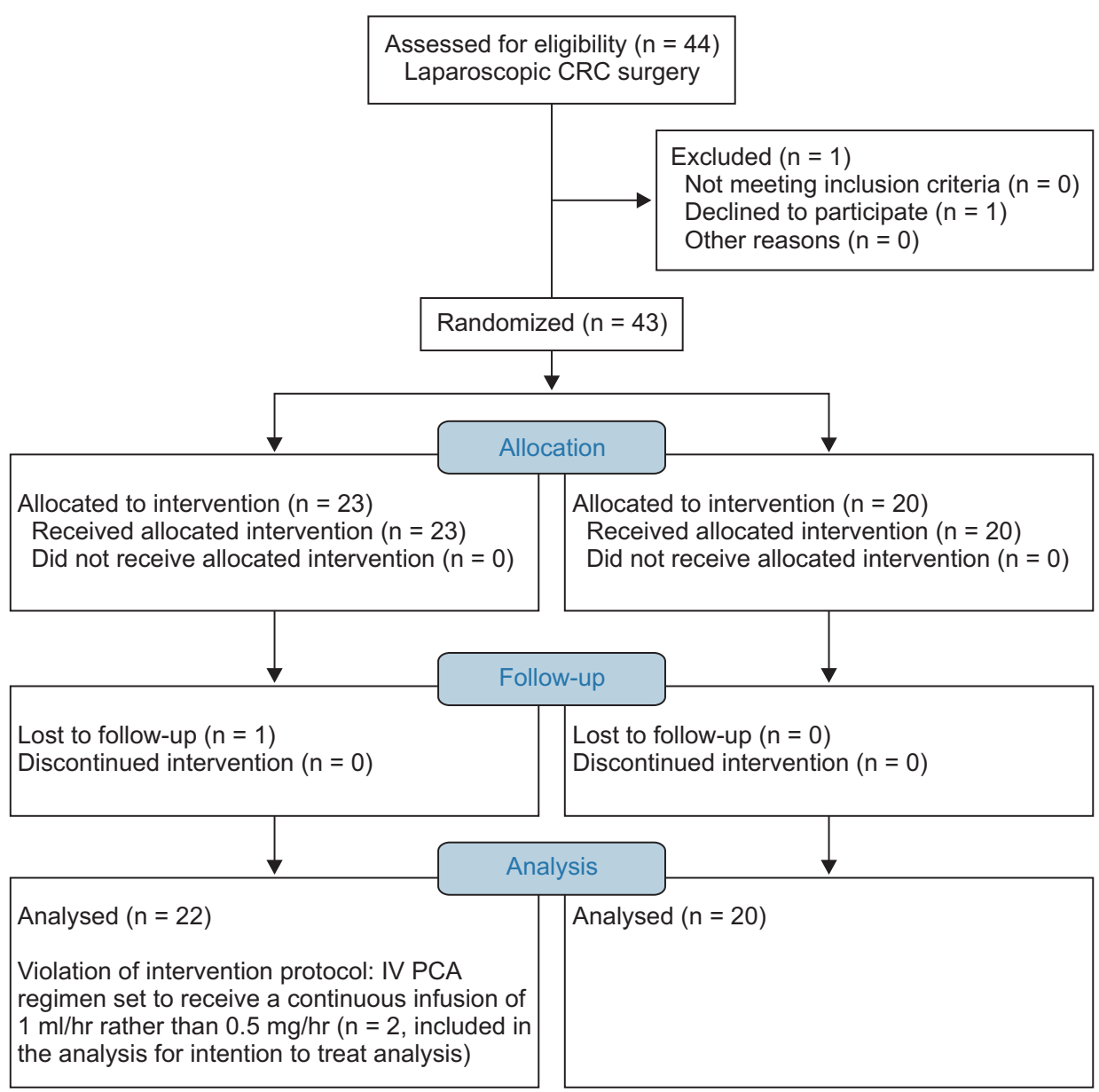

Fig. 2. CONSORT flow diagram. LCRC: laparoscopic colorectal cancer. 
Table 1. Demographic Patient and Surgical Data

\begin{tabular}{|c|c|c|c|}
\hline & $\begin{array}{l}\text { Fentanyl IV PCA } \\
\text { group }(\mathrm{n}=22)\end{array}$ & $\begin{array}{l}\text { Ropivacaine continuous } \\
\text { infusion group }(n=20)\end{array}$ & $\mathrm{P}$ value \\
\hline Age $(y r)$ & $55.3 \pm 7.0$ & $53.4 \pm 8.7$ & 0.605 \\
\hline Gender (M/F) & $14 / 8$ & $12 / 8$ & 0.808 \\
\hline Weight (kg) & $64.5 \pm 10.5$ & $64.0 \pm 11.4$ & 0.554 \\
\hline Height $(\mathrm{cm})$ & $164.4 \pm 7.4$ & $158.3 \pm 19.9$ & 0.296 \\
\hline ASA PS I/II & $12 / 10$ & $14 / 6$ & 0.303 \\
\hline $\mathrm{DM}(\mathrm{Y} / \mathrm{N})$ & $2 / 20$ & $2 / 18$ & 0.920 \\
\hline $\operatorname{HTN}(\mathrm{Y} / \mathrm{N})$ & $8 / 14$ & $5 / 15$ & 0.426 \\
\hline Extraction site of specimen & & & 0.231 \\
\hline Umbilicus/LLQ & $16 / 6$ & $11 / 9$ & \\
\hline Type of laparoscopic surgery (n) & & & 0.438 \\
\hline $\begin{array}{l}\text { Anterior resection/Right hemicolectomy/ } \\
\text { Low anterior resection/Left hemicolectomy }\end{array}$ & $8 / 7 / 7 / 0$ & $11 / 4 / 4 / 1$ & \\
\hline TNM staging (n) I/II/III & $5 / 3 / 14$ & $4 / 5 / 10$ & \\
\hline Crystalloid (ml) & $1106.8 \pm 309.5$ & $1210.0 \pm 307.6$ & 0.090 \\
\hline Colloid (ml) & $181.8 \pm 246.2$ & $75.0 \pm 183.2$ & 0.120 \\
\hline $\mathrm{EBL}(\mathrm{ml})$ & $72.7 \pm 39.3$ & $84.0 \pm 48.3$ & 0.428 \\
\hline Urine output (ml) & $255.7 \pm 140.9$ & $277.5 \pm 167.4$ & 0.938 \\
\hline Duration of anesthesia (min) & $169.6 \pm 32.3$ & $181.9 \pm 40.6$ & 0.338 \\
\hline Duration of surgery (min) & $131.4 \pm 36.3$ & $143.8 \pm 35.9$ & 0.190 \\
\hline Intraoperatively used fentanyl dose $(\mu \mathrm{g})$ & $30.0 \pm 47.0$ & $23.7 \pm 42.0$ & 0.792 \\
\hline
\end{tabular}

Values are mean \pm SD or numbers of patients. IV PCA: Intravenous patient-controlled analgesia, ASA PS: American society of anesthesiologist physical status, DM: diabetes mellitus, HTN: hypertension, LLQ: left lower quadrant, TNM staging: tumor node metastasis staging, EBL: estimated blood loss. $P$ values $<0.05$ are considered statistically significant.

Table 2. The Visual Analogue Scale at Rest and when Coughing during Postoperative Hours

\begin{tabular}{|c|c|c|c|}
\hline & Fentanyl IV PCA group $(\mathrm{n}=22)$ & Ropivacaine continuous infusion group $(\mathrm{n}=20)$ & $P$ value \\
\hline \multicolumn{4}{|l|}{ The VAS at rest } \\
\hline Postoperative $1 \mathrm{~h}$ (at PACU) & $50(30-70)$ & $30(10-65)$ & 0.381 \\
\hline Postoperative $6 \mathrm{~h}$ & $30(20-50)$ & $30(10-30)$ & $<0.999$ \\
\hline Postoperative $24 \mathrm{~h}$ & $30(10-40)$ & $30(17.5-50.0)$ & $<0.999$ \\
\hline Postoperative $48 \mathrm{~h}$ & $20(9-30)$ & $20(10-35)$ & $<0.999$ \\
\hline Postoperative $72 \mathrm{~h}$ & $10(2-20)$ & $17.5(10-30)$ & $<0.999$ \\
\hline \multicolumn{4}{|l|}{ The VAS when coughing } \\
\hline Postoperative $1 \mathrm{~h}$ (at PACU) & $65(40-80)$ & $50(30-80)$ & $<0.999$ \\
\hline Postoperative $6 \mathrm{~h}$ & $65(40-80)$ & $50(30-60)$ & 0.932 \\
\hline Postoperative $24 \mathrm{~h}$ & $50(40-70)$ & $50(50-70)$ & $<0.999$ \\
\hline Postoperative $48 \mathrm{~h}$ & $50(40-60)$ & $50(30-60)$ & $<0.999$ \\
\hline Postoperative $72 \mathrm{~h}$ & $40(30-60)$ & $40(25-50)$ & $<0.999$ \\
\hline
\end{tabular}

Values are median (Q1-Q3). IV PCA: Intravenous patient-controlled analgesia, VAS: visual analogue scale. P values $<0.05$ are considered statistically significant.

Table 3. Comparison of Time to Bowel Recovery, Resumption of Oral Soft Diet, and Hospital Discharge

\begin{tabular}{lccc}
\hline & Fentanyl IV PCA group $(\mathrm{n}=22)$ & Ropivacaine continuous infusion group $(\mathrm{n}=20)$ & P value \\
\hline Time to first flatus $(\mathrm{h})$ & $60.8 \pm 17.9$ & $47.5 \pm 13.9$ & 0.010 \\
Resumption of oral soft diet (days) & $2.5(2-3)$ & $2(2-3)$ & 0.492 \\
Discharge from hospital (days) & $6(5-7)$ & $6(5.0-6.5)$ & 0.853 \\
\hline
\end{tabular}

Values are mean \pm SD or median (Q1-Q3). IV PCA: Intravenous patient-controlled analgesia, PACU: postanesthetic care unit. P values $<0.05$ are considered statistically significant. 
Table 4. Intravenous Patient Controlled Analgesia Fentanyl and Rescue IV Meperidine Consumption during Postoperative Periods

\begin{tabular}{|c|c|c|c|}
\hline & $\begin{array}{l}\text { Fentanyl IV PCA group } \\
\qquad(\mathrm{n}=22)\end{array}$ & $\begin{array}{l}\text { Ropivacaine continuous infusion } \\
\text { group }(\mathrm{n}=20)\end{array}$ & $\mathrm{P}$ value \\
\hline \multicolumn{4}{|c|}{ Cumulative consumption of intravenous fentanyl ( $\mu$ g) (bolus only) } \\
\hline Postoperative $\sim 1 \mathrm{~h}$ (at PACU) & $15.5 \pm 16.2$ & $11.9 \pm 19.5$ & 0.111 \\
\hline Postoperative $\sim 6 \mathrm{~h}$ & $126.8 \pm 71.9$ & $50.6 \pm 36.5$ & $<0.001$ \\
\hline Postoperative $\sim 24 \mathrm{~h}$ & $500.5 \pm 257.3$ & $199.4 \pm 97.9$ & $<0.001$ \\
\hline Postoperative $\sim 48 \mathrm{~h}$ & $850.7 \pm 307.8$ & $363.6 \pm 157.4$ & $<0.001$ \\
\hline Postoperative $\sim 72 \mathrm{~h}$ & $1182.8 \pm 339.0$ & $540.6 \pm 240.6$ & $<0.001$ \\
\hline \multicolumn{4}{|c|}{ Cumulative consumption of rescue intravenous meperidine (mg) } \\
\hline Postoperative $\sim 1 \mathrm{~h}$ (at PACU) & $30.7 \pm 25.0$ & $15.3 \pm 21.7$ & 0.066 \\
\hline Postoperative $\sim 6 \mathrm{~h}$ & $35.2 \pm 30.1$ & $20.3 \pm 23.4$ & 0.159 \\
\hline Postoperative $\sim 24 \mathrm{~h}$ & $76.1 \pm 81.8$ & $42.8 \pm 51.1$ & 0.163 \\
\hline Postoperative $\sim 48 \mathrm{~h}$ & $94.3 \pm 107.1$ & $70.3 \pm 88.8$ & 0.415 \\
\hline Postoperative $\sim 72 \mathrm{~h}$ & $105.7 \pm 125.4$ & $80.3 \pm 103.8$ & 0.328 \\
\hline $\begin{array}{l}\text { Number (\%) of patients with no request for rescue } \\
\text { IV meperidine during postoperative } 72 \text { hours }\end{array}$ & $4 / 22(18.2 \%)$ & $8 / 20(40 \%)$ & 0.175 \\
\hline $\begin{array}{l}\text { Time to first meperidine (rescue meperidine } \\
\text { given at ward) (h) }\end{array}$ & $12.4(0.0-27.0)$ & $0.0(0.0-10.0)$ & 0.061 \\
\hline $\begin{array}{l}\text { Time to first meperidine (rescue meperidine } \\
\text { given at PACU included) }(\mathrm{h})\end{array}$ & $0.4(0.2-22.5)$ & $0.2(0.0-6.3)$ & 0.279 \\
\hline Satisfaction & $(n=9)$ & $(\mathrm{n}=15)$ & \\
\hline Postoperative $0-24 \mathrm{~h}$ & $5.3 \pm 2.3$ & $6.3 \pm 2.4$ & 1.000 \\
\hline Postoperative $24-48 \mathrm{~h}$ & $6.8 \pm 2.0$ & $7.1 \pm 1.8$ & 1.000 \\
\hline Postoperative $48-72 \mathrm{~h}$ & $\begin{array}{c}7.3 \pm 1.5 \\
(\mathrm{n}=8)\end{array}$ & $\begin{array}{l}6.6 \pm 1.7 \\
(\mathrm{n}=11)\end{array}$ & 0.988 \\
\hline
\end{tabular}

$\overline{\text { Values are mean } \pm \text { SD or median }(\mathrm{Q} 1-\mathrm{Q} 3) \text {. IV PCA: Intravenous patient-controlled analgesia, PACU: postanesthetic care unit. P values }<0.05 \text { are }}$ considered statistically significant.

Table 5. Postoperative Adverse Effects

\begin{tabular}{|c|c|c|c|}
\hline & $\begin{array}{l}\text { Fentanyl IV PCA group } \\
\qquad(\mathrm{n}=22)\end{array}$ & $\begin{array}{l}\text { Ropivacaine continuous infusion group } \\
\qquad(\mathrm{n}=20)\end{array}$ & $P$ value \\
\hline \multicolumn{4}{|l|}{ PONV incidence (yes/total) } \\
\hline Postoperative $0-1 \mathrm{~h}$ & $2 / 22$ & $3 / 20$ & 0.555 \\
\hline Postoperative $1-6 \mathrm{~h}$ & $1 / 22$ & $3 / 20$ & 0.249 \\
\hline Postoperative 6-24h & $3 / 22$ & $1 / 20$ & 0.945 \\
\hline Postoperative $24-48 \mathrm{~h}$ & $0 / 22$ & $0 / 20$ & NA \\
\hline Postoperative $48-72 \mathrm{~h}$ & $1 / 22$ & $0 / 20$ & 0.335 \\
\hline \multicolumn{4}{|l|}{ Use of antiemetic drug (yes) } \\
\hline Postoperative $0-1 \mathrm{~h}$ & $2 / 22$ & $1 / 20$ & 0.607 \\
\hline Postoperative $1-6 \mathrm{~h}$ & $11 / 22$ & $5 / 20$ & 0.096 \\
\hline Postoperative 6-24 h & $1 / 22$ & $0 / 20$ & 0.335 \\
\hline Postoperative $24-48 \mathrm{~h}$ & $0 / 22$ & $1 / 20$ & 0.288 \\
\hline Postoperative $48-72 \mathrm{~h}$ & $0 / 22$ & $0 / 20$ & NA \\
\hline \multicolumn{4}{|c|}{ Sedation (1: completely awake, 2 : drowsy, 3 : dozing, 4 : mostly sleepy, 5 : not responding) } \\
\hline Postoperative $0-1 \mathrm{~h}$ & $10 / 8 / 1 / 3 / 0$ & $11 / 8 / 1 / 0 / 0$ & 0.219 \\
\hline Postoperative $1-6 \mathrm{~h}$ & $16 / 5 / 1 / 0 / 0$ & $11 / 8 / 0 / 1 / 0$ & 0.651 \\
\hline Postoperative 6-24 h & $20 / 0 / 2 / 0 / 0$ & $19 / 0 / 0 / 1 / 0$ & 0.489 \\
\hline Postoperative $24-48 \mathrm{~h}$ & $22 / 0 / 0 / 0 / 0$ & $20 / 0 / 0 / 0 / 0$ & NA \\
\hline Postoperative $48-72 \mathrm{~h}$ & $22 / 0 / 0 / 0 / 0$ & $20 / 0 / 0 / 0 / 0$ & NA \\
\hline
\end{tabular}

Values are number of patients. IV PCA: Intravenous patient-controlled analgesia, PONV: postoperative nausea and vomiting. 


\section{Discussion}

This randomized trial compared IV PCA opioid-based analgesic method to continuous local anesthetic-based pain control. Our results showed that analgesia with ropivacaine continuous infusion after LCRC surgery was not different from fentanyl IV PCA. The VAS was not different between the groups throughout the study. Bowel recovery manifested by the time to first flatus was significantly faster in the R group. During the 72 hour follow up, rescue IV meperidine was never requested in 8 out of 20 patients (40\%) in the R group versus 4 out of 22 patients (18.2\%) in the F group, although the percentage of patient requiring meperidine was not significantly different between the groups ( $\mathrm{P}$ $=0.175$ ). Fentanyl IV PCA doses were also less administered in the R group.

Conversely, time to first meperidine request in the ward $(0.0$ $[0.0-10.0]$ hour $)$ was significantly shorter in the $\mathrm{R}$ group $(\mathrm{P}=$ 0.017). However, rescue IV meperidine administration during the 72 hour follow up was less in the R group, without statistical significance. These results signify that patients required rescue IV meperidine immediately upon transfer to the ward. Earlier requests for rescue meperidine, yet smaller rescue meperidine and fentanyl consumption with fast bowel recovery in the $\mathrm{R}$ group may seem contradictory. However, we reason that patients in the $\mathrm{R}$ group needed earlier meperidine immediately after transfer to the ward, because ropivacaine infusion required time to reach peak effect, whereas fentanyl IV PCA had fast onset systemic analgesia in patients in the F group. Furthermore, transfer to the ward may have caused patients to move from bed to bed, and this movement could have made patients in the $\mathrm{R}$ group request rescue meperidine at that timepoint. We therefore think that an earlier request for rescue IV meperidine in the $\mathrm{R}$ group was as a result of different pain control modalities and does not contradict the favorable pain control effect of ropivacaine continuous infusion with smaller meperidine and fentanyl consumption. To overcome an earlier request for IV rescue meperidine, local anesthetics with an earlier onset such as lidocaine bolus administration may be favorable.

In perspectives of ERAS, local anesthetics continuous infusion may be an attractive alternative to systemic opioids or neuraxial block, especially if neuraxial block is not indicated $[9,10,20,23]$, because the former may enable adequate analgesia with early return of bowel function and a shorter hospital stay. However, different studies yield disparate results on ropivacaine wound infusion, with some studies reporting beneficial effects and others reporting no beneficial effects $[21,24,25]$. The discrepancies among the studies were attributed to the anatomical plane of catheter insertion and the dose of local anesthetics used $[9,19,21]$. A meta-analysis by Ventham et al. [9] revealed that subfascial wound catheters display more consistent analgesic ef- fect compared to subcutaneous, suprafascial wound catheters. In a study by Moore et al. [19], the researchers placed the catheter at the neural plane rather than at the muscular plane and it produced more effective analgesia. Proximity to the target nociceptors and nerves was one determining factor for the efficacy of local anesthetics wound infusion [19]. In abdominal surgeries, proximity to the nerves arising from the thoracic intercostal nerves T6-12, L1 may be a rational choice, therefore, placing the catheters between the internal oblique and transversus abdominis muscle at the lateral side or at the preperitoneal space at the midline seems reasonable. In this regard, the TAP is known to be an effective target of nerve blockade in abdominal surgeries $[17,26]$. However, in order to place the catheters at the TAP in LCRC surgery, utilization of ultrasonography is prerequisite. In our study, we instead placed the ropivacaine continuous catheters at the subfascial layer, i.e., the preperitoneal layer between the parietal peritoneum and the musculo-fascial layer, because this was technically feasible without the use of ultrasonography and safe to approach under laparoscopic guidance in LCRC surgery. According to Ventham et al. [9], subfascial catheters can provide effective pain control. We therefore thought that our method could also be an alternative choice for effective pain control.

Until recently, pain management in ERAS has been studied mainly in open abdominal surgeries [27]. As studies on the best methods of pain control in LCRC surgeries emerged, few studies described a local anesthetics wound infusion catheter at the extraction wound of the specimen [12,19]. Moore et al. [19] reported comparable analgesic effect of ropivacaine wound infusion with early improved respiratory function, compared to the placebo. In their study they concluded that wound infusion did not offer improved analgesia or beneficial effect in bowel recovery. Boulind et al.s [12] report was a feasibility study, in which they did not conclude on the efficacy of the wound infusion. However, they strongly suggested that local anesthetic is a potentially strong alternative analgesic method, and that a further large scale double blinded randomized trial was feasible. Our study was different from the two studies in several aspects. Instead of placing a catheter at the wound, we used two catheters on each side of the subcostal margin at the plane close to the peritoneal layer and $0.5 \%$ ropivacaine was infused at $2 \mathrm{ml} /$ h per catheter. In Moore et al.s study [19], they used a single catheter at the extraction wound and used an infusion pump to provide $0.5 \%$ ropivacaine at $8 \mathrm{ml} / \mathrm{h}$ [19]. Their study compared ropivacaine continuous infusion with saline infusion. But our study compared different analgesic modalities, comparing between opioid-based versus local anesthetic-based analgesia. In LCRC surgery, surgical site, laparoscopic port incision, and specimen extraction incision are located in the lower abdomen. Therefore, we chose to target ropivacaine wound infusion along 
the subcostal line at subfascial layer, because we wanted to block the intercostal nerve trajectory at the upper level to cover antinociception not only at the surgical incision, but also to reduce diffuse abdominal pain caused by nociceptive triggering in the whole peritoneum by $\mathrm{CO}_{2}$ gas insufflation during laparoscopy [22]. We cannot extrapolate which plane of catheter insertion is better in pain control between ours or that in the previous studies. However, we suggest that incisional pain and pneumoperitoneum induced pain may be managed better with bilateral subcostal subfascial catheter placement as in our study, rather than at the extraction wound.

Early passage of flatus is an important benefit of local anesthetics continuous infusion [9]. Postoperative ileus delays postoperative recovery, patient discomfort, and hospital discharge. Recovery of bowel function after surgery can be affected by several factors, such as hypoalbuminemia, narcotics, nasogastric tube, and bowel manipulation during surgery [28-30]. In Moore et al.s study [19], they compared ropivacaine wound infusion with placebo and despite less consumption of morphine in ropivacaine infusion group, the bowel recovery was not different between the groups. We speculated that differences in bowel recovery between Moore et al.s study and ours may be explained by ERAS programs implemented by each institution. In the Moore study, they began a fluid diet on postoperative day (POD) 1; whereas in our study, a fluid diet was initiated on POD 2-4, according to ERAS care (Table 6). The ERAS management used in this study (Table 6) included early enteral feeding postoperatively according to surgery type and each patient's bowel condition, regardless of the presence of bowel flatus passage. Nonetheless, reduced use of perioperative opioid is associated with early postoperative bowel recovery, which was in agreement with our findings.

Postoperative adverse effects including PONV and sedation were not different between the groups, and hypotension, diz-

Table 6. Early Recovery after Surgery Care Components Used in this Study

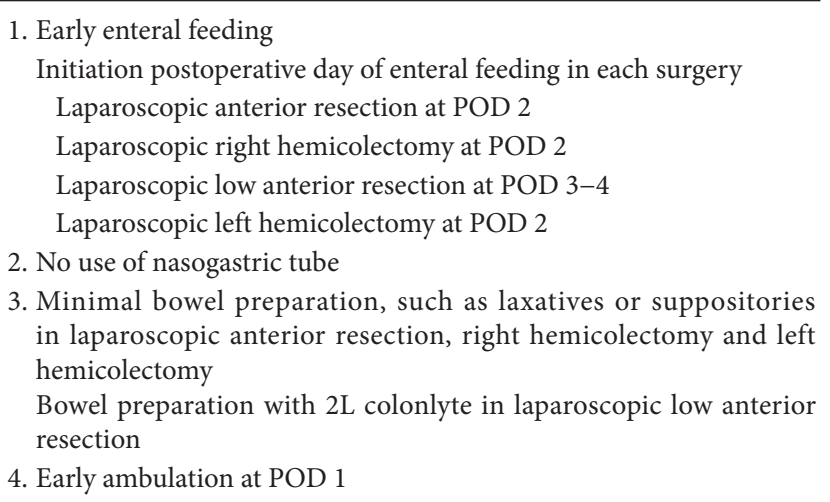

POD: postoperative day.

Online access in http://ekja.org ziness, headache or significant wound complications were not present in this study.

The limitations of our study are the following. First, this study was carried out by non-blinding, because patients in the $\mathrm{R}$ group carried a continuous wound infusion device, but patients in the F group did not. We did not use a placebo (saline) based wound infusion device in the F group to double blind the study because the comparison was between two different modalities of analgesia, i.e., systemic IV opioid based analgesia versus local anesthetics based analgesia. In an effort to reduce the risk of bias in this study, our assessor was not informed of and was oblivious to the presence of a wound device, while investigating the pain scores, side effects, and IV PCA infusion doses. Second, our study was conducted in a single institution and by a single surgeon conducting LCRC surgery, limiting the study's generalizability. Also, the sample size may have been too small to demonstrate the statistical significance in rescue meperidine consumption, although clinically, less meperidine was required in the $\mathrm{R}$ group. A largescale or multicentered investigation needs to be performed to validate our results. Third, long term follow up of patients was not conducted. However, electronic chart review from outpatient follow up after surgery showed no incidence of residual severe pain after surgery. Fourth, the plasma concentration of ropivacaine was not measured. However, there are several other studies that confirm safety and reliable use of $0.5 \%$ ropivacaine at $4 \mathrm{ml} / \mathrm{h}$ in wound infiltration to support its use.

In conclusion, subfascial ropivacaine continuous infusion in LCRC surgeries, along the subcostal area in an inverse " $v$ " shape at the subfascial layer, can provide analgesia not different from fentanyl IV PCA, with early bowel recovery. Earlier requests for meperidine in the ropivacaine infusion group in the ward may be related to the peak effect time of ropivacaine continuous infusion, so this does not contradict the favorable pain control effect of the R group with fewer IV meperidine requests. Further investigations using or comparing this method of ropivacaine continuous infusion at the subfacial layer (preperitoneum) with ropivacaine continuous infusion at the extraction wound may be considered.

\section{Acknowledgments}

There was no other source of funding for research. However, this study received On-Q Painbuster ${ }^{\mathrm{TM}}$ (I-Flow Corp., Lake Forest, CA, USA) product supply from B-Braun corporation, without prejudice nor other financial support. 


\section{References}

1. Vlug MS, Wind J, Hollmann MW, Ubbink DT, Cense HA, Engel AF, et al. Laparoscopy in combination with fast track multimodal management is the best perioperative strategy in patients undergoing colonic surgery: a randomized clinical trial (LAFA-study). Ann Surg 2011; 254: 868-75.

2. Kuhry E, Schwenk W, Gaupset R, Romild U, Bonjer J. Long-term outcome of laparoscopic surgery for colorectal cancer: a cochrane systematic review of randomised controlled trials. Cancer Treat Rev 2008; 34: 498-504.

3. Liang Y, Li G, Chen P, Yu J. Laparoscopic versus open colorectal resection for cancer: a meta-analysis of results of randomized controlled trials on recurrence. Eur J Surg Oncol 2008; 34: 1217-24.

4. Lourenco T, Murray A, Grant A, McKinley A, Krukowski Z, Vale L. Laparoscopic surgery for colorectal cancer: safe and effective? - A systematic review. Surg Endosc 2008; 22: 1146-60.

5. Miller TE, Thacker JK, White WD, Mantyh C, Migaly J, Jin J, et al. Reduced length of hospital stay in colorectal surgery after implementation of an enhanced recovery protocol. Anesth Analg 2014; 118: 1052-61.

6. Levy BF, Tilney HS, Dowson HM, Rockall TA. A systematic review of postoperative analgesia following laparoscopic colorectal surgery. Colorectal Dis 2010; 12: 5-15.

7. Nadler A, Pearsall EA, Victor JC, Aarts MA, Okrainec A, McLeod RS. Understanding surgical residents' postoperative practices and barriers and enablers to the implementation of an Enhanced Recovery After Surgery (ERAS) Guideline. J Surg Educ 2014; 71: 632-8.

8. Joshi GP, Bonnet F, Kehlet H. Evidence-based postoperative pain management after laparoscopic colorectal surgery. Colorectal Dis 2013; 15: 146-55.

9. Ventham NT, O'Neill S, Johns N, Brady RR, Fearon KC. Evaluation of novel local anesthetic wound infiltration techniques for postoperative pain following colorectal resection surgery: a meta-analysis. Dis Colon Rectum 2014; 57: 237-50.

10. Wongyingsinn M, Baldini G, Charlebois P, Liberman S, Stein B, Carli F. Intravenous lidocaine versus thoracic epidural analgesia: a randomized controlled trial in patients undergoing laparoscopic colorectal surgery using an enhanced recovery program. Reg Anesth Pain Med 2011; 36: 241-8.

11. Levy BF, Scott MJ, Fawcett W, Fry C, Rockall TA. Randomized clinical trial of epidural, spinal or patient-controlled analgesia for patients undergoing laparoscopic colorectal surgery. Br J Surg 2011; 98: 1068-78.

12. Boulind CE, Ewings P, Bulley SH, Reid JM, Jenkins JT, Blazeby JM, et al. Feasibility study of analgesia via epidural versus continuous wound infusion after laparoscopic colorectal resection. Br J Surg 2013; 100: 395-402.

13. Lee C, Song YK. The effect of intraperitoneal instillation and trocar site infiltration of $0.25 \%$ levobupivacaine on the postoperative pain after performing laparoscopic cholecystectomy under remifentanil based anesthesia. Korean J Pain 2008; 21: 44-50.

14. Hübner M, Blanc C, Roulin D, Winiker M, Gander S, Demartines N. Randomized clinical trial on epidural versus patient-controlled analgesia for laparoscopic colorectal surgery within an enhanced recovery pathway. Ann Surg 2015; 261: 648-53.

15. Halabi WJ, Kang CY, Nguyen VQ, Carmichael JC, Mills S, Stamos MJ, et al. Epidural analgesia in laparoscopic colorectal surgery: a nationwide analysis of use and outcomes. JAMA Surg 2014; 149: 130-6.

16. Ventham NT, Hughes M, O'Neill S, Johns N, Brady RR, Wigmore SJ. Systematic review and meta-analysis of continuous local anaesthetic wound infiltration versus epidural analgesia for postoperative pain following abdominal surgery. Br J Surg 2013; 100: 1280-9.

17. Niraj G, Kelkar A, Hart E, Horst C, Malik D, Yeow C, et al. Comparison of analgesic efficacy of four-quadrant transversus abdominis plane (TAP) block and continuous posterior TAP analgesia with epidural analgesia in patients undergoing laparoscopic colorectal surgery: an open-label, randomised, non-inferiority trial. Anaesthesia 2014; 69: 348-55.

18. Braga M, Vignali A, Zuliani W, Radaelli G, Gianotti L, Martani C, et al. Metabolic and functional results after laparoscopic colorectal surgery: a randomized, controlled trial. Dis Colon Rectum 2002; 45: 1070-7.

19. Moore SE, Reid KC, Draganic BD, Smith SR. Randomized clinical trial of ropivacaine wound infusion following laparoscopic colorectal surgery. Tech Coloproctol 2012; 16: 431-6.

20. Gupta A, Favaios S, Perniola A, Magnuson A, Berggren L. A meta-analysis of the efficacy of wound catheters for post-operative pain management. Acta Anaesthesiol Scand 2011; 55: 785-96.

21. Bertoglio S, Fabiani F, Negri PD, Corcione A, Merlo DF, Cafiero F, et al. The postoperative analgesic efficacy of preperitoneal continuous wound infusion compared to epidural continuous infusion with local anesthetics after colorectal cancer surgery: a randomized controlled multicenter study. Anesth Analg 2012; 115: 1442-50.

22. Fredman B, Jedeikin R, Olsfanger D, Flor P, Gruzman A. Residual pneumoperitoneum: a cause of postoperative pain after laparoscopic cholecystectomy. Anesth Analg 1994; 79: 152-4.

23. Thornton PC, Buggy DJ. Local anaesthetic wound infusion for acute postoperative pain: a viable option? Br J Anaesth 2011; 107: 656-8.

24. Polglase AL, McMurrick PJ, Simpson PJ, Wale RJ, Carne PW, Johnson W, et al. Continuous wound infusion of local anesthetic for the control of pain after elective abdominal colorectal surgery. Dis Colon Rectum 2007; 50: 2158-67.

25. Forastiere E, Sofra M, Giannarelli D, Fabrizi L, Simone G. Effectiveness of continuous wound infusion of $0.5 \%$ ropivacaine by On-Q pain relief system for postoperative pain management after open nephrectomy. Br J Anaesth 2008; 101: 841-7.

26. Conaghan P, Maxwell-Armstrong C, Bedforth N, Gornall C, Baxendale B, Hong LL, et al. Efficacy of transversus abdominis plane blocks in 
laparoscopic colorectal resections. Surg Endosc 2010; 24: 2480-4.

27. Hughes MJ, Ventham NT, McNally S, Harrison E, Wigmore S. Analgesia after open abdominal surgery in the setting of enhanced recovery surgery: a systematic review and meta-analysis. JAMA Surg 2014; 149: 1224-30.

28. Ay AA, Kutun S, Ulucanlar H, Tarcan O, Demir A, Cetin A. Risk factors for postoperative ileus. J Korean Surg Soc 2011; 81: 242-9.

29. Woods MS, Kelley H. Oncotic pressure, albumin and ileus: the effect of albumin replacement on postoperative ileus. Am Surg 1993; 59: 758-63.

30. Kalff JC, Schraut WH, Simmons RL, Bauer AJ. Surgical manipulation of the gut elicits an intestinal muscularis inflammatory response resulting in postsurgical ileus. Ann Surg 1998; 228: 652-63. 